\title{
PROPIEDADES PSICOMÉTRICAS DE LA ESCALA GENCAT DE CALIDAD DE VIDA EN UNA MUESTRA DE PERSONAS SIN HOGAR
}

\section{Gencat Psychometric Properties of Scale of Quality of Life in a Sample of Homeless}

\author{
Eva María Benito Herráez \\ Universidad Nacional de Educación a Distancia (UNED). Facultad de Psicología. C/ Juan del \\ Rosal, 4. 28040 Madrid (España) \\ evabenito@pip.udl.cat; ebenito@paeria.es \\ Carles Alsinet Mora \\ Universitat de Lleida. Departamento de Pedagogía y Psicología \\ Araceli MACIÁ ANTÓN \\ Universidad Nacional de Educación a Distancia (UNED). Facultad de Psicología. Cl Juan del \\ Rosal, 4. 28040 Madrid (España)
}

Recepción: 2 de junio de 2015

Fecha de aceptación definitiva: 21 de enero de 2016

Resumen: El objetivo principal de esta investigación es analizar las propiedades psicométricas de la Escala Gencat de Calidad de Vida en una muestra de 72 personas usuarias de la Oficina Municipal de Atención para Personas en Situación de sin Hogar de la ciudad de Lleida. El análisis de los 69 ítems se ha basado en la Teoría Clásica de los Test y en la Teoría de Respuesta al Ítem. Los resultados del alfa de Cronbach muestran valores adecuados, situándose entre .749 y .853. Según el modelo de Escalas de Clasificación la confiabilidad es alta con valores superiores a .95 en todas las dimensiones. La mayoría de ítems se ajusta al modelo. La limitación más importante del estudio es el tamaño de la muestra, que disminuye su confiabilidad. A pesar de ello se puede concluir que la escala posee evidencias suficientes de fiabilidad para poder evaluar la calidad de vida de las personas sin hogar.

Palabras Clave: calidad de vida; personas sin hogar; teoría de respuesta al ítem. 
PROPIEDADES PSICOMÉTRICAS DE LA ESCALA GENCAT DE CALIDAD DE VIDA

EN UNA MUESTRA DE PERSONAS SIN HOGAR

EVA MARÍA BENITO HERRÁEZ, CARLES ALSINET MORA Y ARACELI MACIÁ ANTÓN

ABSTRACT: The main aim of this research is to analyse the psychometric properties of the Scale Gencat of Quality of Life in a sample of 72 people users of the Municipal Office of Attention for homeless people in the city of Lleida. The analysis of the 69 items was based on Classical Test Theory and in the Item Response Theory. The results obtained with Cronbach's alpha show appropiate values ranging between .749 and .853 . According to the Rating Scale Model reliability is high, with values higher than .95 in all dimensions. The majority of items fit the model. The most important limitation in the study is the sample size, which reduced its reliability. Despite this, it can be concluded that the scale has sufficient evidence of reliability to evaluate the quality of life for homeless.

KEY WORDS: quality of life; homeless; item response theory.

\section{Introducción}

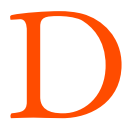

EFINIR CALIDAD DE VIDA (CV) no es tarea fácil. Históricamente, el concepto ha sufrido modificaciones y cambios desde sus inicios en la filosofía hasta constructos basados en criterios empíricos. La concreción de su significado ha sido y sigue siendo una labor compleja.

Sin embargo y a pesar de las dificultades de conceptualización existe un cierto acuerdo en relación a los aspectos básicos de su definición. Tal y como concluyen Cummins y Cahill (2000) al revisar la evolución del constructo de CV existen ciertos elementos aceptados por la comunidad científica. Es un concepto multidimensional formado por diferentes ámbitos de la vida de la persona, que incluye componentes objetivos y subjetivos, aplicable a cualquiera independientemente de sus circunstancias vitales y ha de reflejar las normas culturales de bienestar objetivo. Teniendo en cuenta los elementos comunes han surgido una serie de modelos explicativos que se centran en determinados factores o dimensiones, como los de calidad de vida relacionada con la salud (CVRS) o calidad de vida familiar (CVF), que deben ser diferenciados del de la CV individual.

La aparición del constructo de CVRS se produce a partir de la definición que la Organización Mundial de la Salud (OMS) hizo en 1948 acerca de la salud, considerándola como un estado de completo bienestar físico, psíquico y social y no únicamente la ausencia de enfermedad. Esta definición es fundamental en el desarrollo de la CV, que a su vez está estrechamente relacionada con la CVRS. Schumaker y Naughton (1995) determinan que está compuesta por el estado funcional, el funcionamiento psicológico y social y los síntomas que provoca el problema de salud que padezca la persona y su tratamiento. Una década más tarde, Schwartzmann (2003) plantea que la CVRS es la valoración que realiza una persona de acuerdo con sus propios criterios del estado físico, emocional y social en que se encuentra y refleja el grado de satisfacción con una situación personal a nivel fisiológico, emocional y social.

Para Schalock, Brown, Brown, Cummins, Felce, Matikka, Keith y Parmenter (2002) la CV es un constructo multidimensional que está afectado por factores personales y ambientales, siendo la vida familiar uno de ellos. Según Park, Turnbull y Turnbull (2002) la familia experimenta CV cuando cada uno de sus miembros tiene 
sus necesidades cubiertas, disfrutan de la vida juntos y tienen las oportunidades precisas para conseguir y alcanzar las metas importantes para ellos. La CVF es entendida por numerosos autores como una extensión de la CV individual (CórdobaAndrade, Gómez-Benito y Verdugo-Alonso, 2008).

Estos dos modelos anteriores se centran en dimensiones específicas del constructo. Por el contrario, la CV individual supone una perspectiva más amplia que incluye todas las dimensiones relevantes en la vida de una persona e incluye la evaluación de elementos objetivos y subjetivos, aunque son estos últimos uno de sus elementos clave (Schalock y Verdugo, 2003). Entre los modelos de CV individual destacan el de Cummins (1997, 2000), quien recoge en su definición tanto componentes objetivos como subjetivos, incorporando éstos medidas de bienestar percibido, y el modelo de Felce y Perry (1995), que ofrece tres componentes de la CV que interactúan entre sí: las condiciones de vida objetivas, la evaluación subjetiva de la satisfacción personal y los valores personales. El modelo fue adaptado con posterioridad por Petry, Maes y Vlaskamp (2005) en personas con discapacidad profunda y múltiple.

Dentro del ámbito de la discapacidad es claramente el modelo de CV de Schalock y Verdugo el que ha exhibido un desarrollo más evidente y una amplia aceptación a nivel internacional (Gómez, Verdugo, Arias y Arias, 2010). Considera la CV individual como un fenómeno subjetivo basado en la percepción que tiene una persona de varios aspectos de las experiencias de su vida incluyendo las características personales, condiciones objetivas de vida y las percepciones de los otros significativos. Los autores consideran la CV como un concepto multidimensional compuesto por las mismas ocho dimensiones para todas las personas: el Bienestar Físico, el Bienestar Emocional, las Relaciones Interpersonales, la Inclusión Social, el Desarrollo Personal, el Bienestar Material, la Autodeterminación y los Derechos. Estas dimensiones se operacionalizan mediante indicadores de CV entendidos como las percepciones, comportamientos y condiciones relacionadas con la CV que aportan una indicación sobre el bienestar de una persona y son una base conceptual y empírica para la medición y la aplicación del concepto, así como para la evaluación de los resultados personales, que reflejan la situación personal en relación a los indicadores planteados.

Los autores incorporan al modelo la perspectiva de sistemas al proponer que tanto las dimensiones como sus indicadores se estructuran dentro de un sistema triple: el microsistema o contexto social inmediato; el mesosistema compuesto por la comunidad, los grupos y organizaciones que afectan el funcionamiento del microsistema, y el macrosistema, que serían los patrones culturales, el sistema económico y otros factores sociales que influyen en los valores y creencias de la sociedad. Estos tres sistemas tienen influencia directa en la evaluación de la CV.

Tal y como puntualizan Gómez, Verdugo y Arias (2010) una de las aportaciones más importantes del modelo se encuentra en la evaluación y mejora de los resultados personales relacionados con la CV de los destinatarios de la intervención. Para llevar a cabo esta evaluación, los instrumentos de medición son fundamentales, ya que la $\mathrm{CV}$ se ha convertido en un constructo medible que puede aportar información a los responsables de gestionar servicios y apoyos para personas con discapacidad intelectual, siendo extensibles también a otros colectivos que necesiten determinados 
apoyos (Schalock y Verdugo, 2007). Ejemplo de ello serían los destinados a personas con discapacidad intelectual como la Escala Integral (Verdugo, Arias, Gómez y Schalock, 2009) y la Escala INICO-FEAPS (Verdugo, Gómez, Arias, Santamaría, Clavero y Tamarit, 2013), o a personas mayores como la Escala FUMAT de Calidad de Vida (Verdugo, Gómez y Arias, 2009).

La extensión del modelo de CV a otros colectivos se manifiesta claramente en el desarrollo de la Escala Gencat de Calidad de Vida (Verdugo, Arias, Gómez y Schalock, 2008). Es un instrumento de evaluación objetiva diseñado a partir del modelo multidimensional de CV propuesto por Schalock y Verdugo (2003). Siguiendo a Gómez (2010) el instrumento pretende la mejora continua de los servicios sociales, permitiendo el desarrollo e implementación de planes de atención y apoyo individualizados de las personas usuarias siempre basados en resultados personales medibles. Para conseguirlo, presenta un conjunto de aspectos observables de las diferentes dimensiones de CV de la persona, que deben ser evaluados por el profesional de servicios sociales para conformar estos planes de apoyo. Según Verdugo, Arias, Gómez y Schalock (2010), cuando se pretende conseguir cambios organizativos, evaluar programas o mejorar la calidad del servicio es recomendable la utilización de cuestionarios objetivos basados en la observación directa de experiencias y situaciones personales, como lo es la escala Gencat.

Pese a no ser un instrumento específicamente diseñado para la medida de CV de personas sin hogar (PSH), empiezan a aparecer tímidamente experiencias de aplicación de la escala al colectivo. Entre las más destacables se encuentra la desarrollada por Ruiz, Verdugo y Orgaz (2011), quienes analizan la valoración objetiva de la CV de trabajadores con discapacidad o en situación de exclusión social participantes en un programa nacional de empleo con apoyo. La pertinencia de la escala así como sus posibilidades de aplicación e idoneidad para medir la CV del colectivo de PSH se hacen evidentes en estudios como el de Aguirre (2013), quien utiliza los resultados obtenidos para planificar intervenciones específicas y adaptadas a las necesidades de los usuarios de servicios sociales destinados a este grupo de población. Partiendo de esta premisa, el principal objetivo de la presente investigación es comprobar si posee adecuadas propiedades psicométricas para poder ser aplicada al colectivo de PSH, al no encontrarse estudios similares. Se ha analizado la fiabilidad mediante la Teoría Clásica de los Test (TCC) y la Teoría de Respuesta al Ítem (TRI), seleccionando para ello la extensión del Modelo de Rasch para ítems politómicos, el Modelo de Escalas de Clasificación (RSM).

\section{Método}

\section{Participantes}

Han participado un total de 72 personas usuarias de la Oficina Municipal de Atención Social para PSH de la ciudad de Lleida: 61 hombres y 11 mujeres, con edades comprendidas entre los 25 y los 73 años. Los resultados muestran que el 
$84.7 \%$ del total son hombres frente al $15.3 \%$ de mujeres, la edad media es de 45 años $(d t=10.84)$, solteros, sin estudios o con estudios primarios y que se encuentran en situación de calle desde hace seis años y medio $(d t=6.84)$. Las personas de origen español suponen el $38.9 \%$ de la muestra, seguidas de las de origen marroquí con un $18 \%$ del total, mientras que el $7 \%$ son nativos de Ghana y Rumanía en ambos casos. El estudio de la normalidad muestra un buen ajuste en la variable edad confirmado mediante los resultados del test de Kolmogorov-Smirnov $(p=.200)$ y Shapiro-Wilks $(p=.366)$. Sin embargo, la variable tiempo en calle manifiesta una desviación significativa ( $p=.000$ en ambos estadísticos). El test de Levene pone de manifiesto la homogeneidad de sus varianzas ( $p=.464$ y .617 respectivamente).

El único criterio de inclusión utilizado en el estudio ha sido la vinculación previa de los participantes por un periodo mínimo de tres meses a la Oficina Municipal según se establece en los requisitos de la escala. En cuanto a los profesionales del equipo de Atención Social para PSH de la ciudad de Lleida encargados de cumplimentar la escala han participado los cuatro componentes el equipo: dos educadores sociales y dos trabajadoras sociales. Cada uno ha sido responsable de cumplimentar la escala relativa a aquellas personas en seguimiento e intervención por parte de cada profesional por un mínimo de 3 meses, tal y como establece el instrumento.

\section{Instrumento de recogida de datos. Escala Gencat de Calidad de Vida}

Es un cuestionario compuesto por 69 ítems relativos a cuestiones observables con la CV individual y distribuidos en ocho subescalas correspondientes a cada dimensión del modelo multidimensional de CV de Schalock y Verdugo: Bienestar Físico, Bienestar Emocional, Relaciones Interpersonales, Inclusión Social, Desarrollo Personal, Bienestar Material, Autodeterminación y Derechos. Los ítems están redactados en tercera persona, con formato declarativo y las respuestas se ofrecen en una escala de frecuencia de cuatro opciones ('nunca o casi nunca', 'a veces', 'frecuentemente' y 'siempre o casi siempre'). La escala ofrece el perfil de CV obtenido a partir de puntuaciones estándar de cada una de las ocho dimensiones analizadas y el índice de CV obtenido a partir de la puntuación estándar compuesta calculada a partir de las ocho subescalas. Es el profesional de servicios sociales quien administra el cuestionario a personas mayores de 18 años conocidas por éste por un tiempo mínimo de tres meses. En el estudio se ha utilizado la versión de la escala en catalán, idioma utilizado por los profesionales que han cumplimentado la escala.

La escala Gencat ha sido desarrollada mediante un método riguroso y sistemático y se han analizado sus propiedades psicométricas con resultados muy aceptables (Arias, Gómez, Verdugo y Navas, 2010; Gómez, Arias, Verdugo y Navas, 2012a; Verdugo et al., 2008; Verdugo et al., 2010). Los resultados basados en la TCC utilizando para ello el alfa de Cronbach para la muestra manifiestan que la fiabilidad total de la Escala Gencat de Calidad de Vida es $\alpha=.956$. Los resultados por dimensiones son los siguientes: Bienestar Emocional $\alpha=.852$; Relaciones Interpersonales $\alpha=.820$; Bienestar Material $\alpha=.810$; Desarrollo Personal $\alpha=.853$; Bienestar Físico 
$\alpha=.767$; Autodeterminación $\alpha=.691$; Inclusión Social $\alpha=.749$, y Derechos $\alpha=.751$, siendo resultados aceptables. La fiabilidad de la escala mejora sensiblemente la obtenida por el estudio de Verdugo et al. (2008), superándola también en siete de ellas, siendo especialmente importantes las diferencias en Bienestar Físico, Relaciones Interpersonales, Inclusión Social y Bienestar Material y mínimas en Bienestar Emocional. Sin embargo, Autodeterminación obtiene un índice bastante menor.

\section{Procedimiento}

Durante el mes de marzo de 2014 se procedió a explicar las características y proceso de cumplimentación de la escala Gencat a los profesionales. A lo largo de las siguientes semanas se completaron 72 escalas, siendo 18 la media por profesional. Es preciso destacar que se dispone de consentimiento informado de cada uno de los usuarios destinatarios de la evaluación y que a lo largo de la investigación se han respetado los requisitos y estándares éticos. Los resultados obtenidos se analizaron mediante los programas estadísticos necesarios para ello y se informó del resultado a los profesionales a lo largo de octubre de 2014, con el objetivo de disponer de la información necesaria para planificar la intervención social de forma individualizada.

\section{Análisis de los datos}

Una vez recogidos los cuestionarios se procedió al análisis de los resultados utilizando para ello los programas SPSS 17 y Ministep Versión 3.81.0. Los estadísticos utilizados se han basado en la TCT, concretamente el alfa de Cronbach, para ofrecer resultados previos acerca de su fiabilidad y en la TRI. Uno de los modelos de la TRI es el modelo matemático de Rasch. Una extensión de éste, empleada para analizar datos de escalas tipo Likert, es el Rating Scale Model (RSM) o Modelo de Escalas de Clasificación. Uno de sus elementos clave es la importancia del ajuste de los datos a un modelo diseñado previamente y se comprueba mediante el test de ajuste global, de los ítems y de los sujetos utilizando para ello diferentes estadísticos. Además permite comprobar si la escala identifica el rasgo latente, en este caso la CV, de los participantes.

\section{Resultados}

El estudio de la escala Gencat utilizando el modelo de escalas de clasificación permite comprobar si los datos obtenidos se ajustan a la predicción teórica propuesta por el modelo o no.

En las siguientes tablas se ofrecen los resultados medidos a través de los estadísticos de nivel de dificultad en logits, los errores estándar y las medidas de ajuste interno y externo al modelo (infit y outfit respectivamente) para el test, los ítems y las personas. 
PROPIEDADES PSICOMÉTRICAS DE LA ESCALA GENCAT DE CALIDAD DE VIDA

EN UNA MUESTRA DE PERSONAS SIN HOGAR

EVA MARÍA BENITO HERRÁEZ, CARLES ALSINET MORA Y ARACELI MACIÁ ANTÓN

\begin{tabular}{|l|l|c|c|c|c|c|c|c|c|}
\hline \multicolumn{8}{|c|}{ TABLA 1. Resumen del ajuste de los ítems y las personas al modelo } \\
\hline \multirow{2}{*}{} & \multicolumn{2}{|c|}{ Medida (logits) } & \multicolumn{2}{c|}{ Error estándar } & \multicolumn{2}{|c|}{ Infit } & \multicolumn{2}{c|}{ Outfit } \\
\cline { 2 - 11 } & Mínimo & Máximo & Mínimo & Máximo & Mínimo & Máximo & Mínimo & Máximo \\
\hline \multirow{2}{*}{ Personas } & Media & -1.49 & 2.61 & 0.45 & 0.73 & 0.73 & 1.02 & 0.95 & 1.09 \\
\cline { 2 - 11 } & $\begin{array}{l}\text { Desviación } \\
\text { estándar }\end{array}$ & 0.81 & 1.62 & 0.11 & 0.50 & 0.50 & 0.83 & 0.49 & 1.57 \\
\hline \multirow{3}{*}{ Ítems } & Media & 0.0 & 0.0 & 0.15 & 0.35 & 0.96 & 1.28 & 0.95 & 1.09 \\
\cline { 2 - 10 } & $\begin{array}{l}\text { Desviación } \\
\text { estándar }\end{array}$ & 0.77 & 1.92 & 0.01 & 0.22 & 0.21 & 0.67 & 0.23 & 0.58 \\
\hline
\end{tabular}

Tabla 2. Ajuste de los ítems al modelo para cada una de las dimensiones

\begin{tabular}{|l|c|c|c|c|}
\hline BIENESTAR EMOCIONAL & $\begin{array}{c}\text { Medida } \\
\text { (logits) }\end{array}$ & $\begin{array}{c}\text { Error } \\
\text { estándar }\end{array}$ & Infit & Outfit \\
\hline 1. Se muestra satisfecho con su vida presente. & 1.77 & .21 & 0.96 & 0.88 \\
\hline 2. Presenta síntomas de depresión. & -1.53 & .18 & 0.86 & 0.89 \\
\hline 3. Está alegre y de buen humor. & 0.43 & .18 & 0.48 & 0.59 \\
\hline 4. Muestra sentimientos de incapacidad o inseguridad. & -1.01 & .18 & 1.11 & 1.12 \\
\hline 5. Presenta síntomas de ansiedad. & -1.30 & .18 & 1.03 & 0.98 \\
\hline 6. Se muestra satisfecho consigo mismo. & 0.76 & .18 & 0.95 & 0.91 \\
\hline 7. Tiene problemas de comportamiento. & -0.69 & .18 & 1.50 & 1.47 \\
\hline 8. Se muestra motivado a la hora de realizar algún tipo de actividad. & 1.57 & .20 & 1.08 & 1.04 \\
\hline $\begin{array}{l}\text { RELACIONES INTERPERSONALES } \\
\text { 9. Realiza actividades que le gustan con otras personas. }\end{array}$ & $\begin{array}{c}\text { Medida } \\
\text { (logits) }\end{array}$ & $\begin{array}{c}\text { Error } \\
\text { estándar }\end{array}$ & Infit & 0 Outfit \\
\hline 10. Mantiene con su familia la relación que desea. & 1.13 & .21 & 0.75 & 0.75 \\
\hline 11. Se queja de la falta de amigos estables. & 2.18 & .29 & 0.86 & 0.72 \\
\hline 12. Valora negativamente sus relaciones de amistad. & -2.50 & .18 & 0.81 & 0.80 \\
\hline 13. Manifiesta sentirse infravalorado por su familia. & -2.03 & .17 & 0.95 & 0.92 \\
\hline 14. Tiene dificultades para iniciar una relación de pareja. & -1.49 & .16 & 1.13 & 1.10 \\
\hline 15. Mantiene una buena relación con sus compañeros de trabajo. & 0.13 & .17 & 1.45 & 1.30 \\
\hline 16. Manifiesta sentirse querido por las personas importantes para él. & -0.49 & .16 & 0.70 & 0.72 \\
\hline $\begin{array}{l}\text { 17. La mayoría de las personas con las que interactúa tienen una condición } \\
\text { similar a la suya. }\end{array}$ & 0.89 & .20 & 0.76 & 0.71 \\
\hline 18. Tiene una vida sexual satisfactoria. & 0.67 & .19 & 1.18 & 1.61 \\
\hline $\begin{array}{l}\text { BIENESTAR MATERIAL } \\
\text { 19. El lugar donde vive le impide llevar un estilo de vida saludable (ruidos, } \\
\text { humos, olores, oscuridad, escasa ventilación, desperfectos, inaccesibi- } \\
\text { lidad...) }\end{array}$ & -.041 & .15 & 0.68 & 0.64 \\
\hline
\end{tabular}

() Ediciones Universidad de Salamanca / CC BY-NC-ND

Siglo Cero, vol. 47 (1), n. ${ }^{\circ}$ 257, 2016, enero-marzo, pp. 23-36 
PROPIEDADES PSICOMÉTRICAS DE LA ESCALA GENCAT DE CALIDAD DE VIDA

EN UNA MUESTRA DE PERSONAS SIN HOGAR

EVA MARÍA BENITO HERRÁEZ, CARLES ALSINET MORA Y ARACELI MACIÁ ANTÓN

Tabla 2. Ajuste de los ítems al modelo para cada una de las dimensiones (cont.)

20. El lugar donde trabaja cumple con las normas de seguridad.

21. Dispone de los bienes materiales que necesita.

22. Se muestra descontento con el lugar donde vive.

23. El lugar donde vive está limpio.

24. Dispone de los recursos económicos necesarios para cubrir sus necesidades básicas.

25. Sus ingresos son insuficientes para permitirle acceder a caprichos.

26. El lugar donde vive está adaptado a sus necesidades.

\section{DESARROLLO PERSONAL}

27. Muestra dificultad para adaptarse a las situaciones que se le presentan.

28. Tiene acceso a nuevas tecnologías (Internet, teléfono móvil, etc.).

29. El trabajo que desempeña le permite el aprendizaje de nuevas habilidades.

30. Muestra dificultades para resolver con eficacia los problemas que se le plantean.

31. Desarrolla su trabajo de manera competente y responsable.

32. El servicio al que acude toma en consideración su desarrollo personal y aprendizaje de habilidades nuevas.

33. Participa en la elaboración de su programa individual.

34. Se muestra desmotivado en su trabajo.

\section{BIENESTAR FÍSICO}

35. Tiene problemas de sueño.

36. Dispone de ayudas técnicas si las necesita.

37. Sus hábitos de alimentación son saludables.

38. Su estado de salud le permite llevar una actividad normal.

39. Tiene un buen aseo personal.

40. En el servicio al que acude se supervisa la medicación que toma.

41. Sus problemas de salud le producen dolor y malestar.

42. Tiene dificultades de acceso a recursos de atención sanitaria (atención preventiva, general, a domicilio, hospitalaria, etc.).

\section{AUTODETERMINACIÓN}

43. Tiene metas, objetivos e intereses personales.

44. Elige cómo pasar su tiempo libre.

45. En el servicio al que acude tienen en cuenta sus preferencias.

46. Defiende sus ideas y opiniones.

47. Otras personas deciden sobre su vida personal.

48. Otras personas deciden cómo gastar su dinero.

49. Otras personas deciden la hora a la que se acuesta.

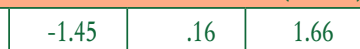

1.41

0.70

\begin{tabular}{c|c|c|c|}
0.92 & .20 & 0.65 & 0.70 \\
\hline-0.80 & .15 & 1.00 & 1.21 \\
\hline-0.67 & .15 & 0.55 & 0.60
\end{tabular}

i- 0.84

\begin{tabular}{l|l|l|l}
0.84 & .20 & 1.12 & 1.00 \\
\hline
\end{tabular}

1.29

0.29

.23

.17

\begin{tabular}{l|l}
1.87 & 1.51 \\
\hline
\end{tabular}

Medida

(logits)

Error

$0.92 \quad 1.08$

$-0.71$

estándar

Infit Outfit

1.36

\begin{tabular}{l|l|l|l}
1.36 & .21 & 0.89 & 1.10 \\
\hline
\end{tabular}

\begin{tabular}{|c|c|c|c|}
\hline-0.52 & .17 & 1.01 & 1.03 \\
\hline 0.13 & .17 & 0.56 & 0.52 \\
\hline-1.12 & .17 & 1.73 & 1.54 \\
\hline 0.35 & .18 & 0.82 & 1.03 \\
\hline-0.71 & .16 & 1.04 & 0.97 \\
\hline Medida & Error & Infit & Outfit \\
\hline
\end{tabular}
(logits)

estándar

$0.88 \quad 1.11$

\begin{tabular}{l|l|l|l|}
-0.78 & .15 & 0.88 & 1.11 \\
\hline-0.63 & .14 & 1.12 & 1.01
\end{tabular}

\begin{tabular}{l|l|l|l|}
-0.63 & .14 & 1.12 & 1.01 \\
\hline 0.73 & .16 & 0.51 & 0.51
\end{tabular}

\begin{tabular}{l|l|l|l|}
\hline 0.73 & .16 & 0.51 & 0.51 \\
\hline
\end{tabular}

\begin{tabular}{|r|r|r|r|}
\hline 0.73 & .16 & 0.51 & 0.51 \\
\hline-0.10 & .17 & 0.84 & 0.85 \\
\hline 0.94 & .14 & 0.86 & 0.77 \\
\hline
\end{tabular}

\begin{tabular}{|c|c|c|c|}
\hline 0.94 & .16 & 2.30 & 1.99 \\
\hline 0.32 & .15 & 1.05 & 1.04 \\
\hline-1.49 & .16 & 0.56 & 0.49 \\
\hline
\end{tabular}

\begin{tabular}{|c|c|c|c|}
\hline-1.49 & .16 & 0.56 & 0.49 \\
\hline $\begin{array}{c}\text { Medida } \\
\text { (logits) }\end{array}$ & $\begin{array}{c}\text { Error } \\
\text { estándar }\end{array}$ & Infit & Outfit \\
\hline 0.88 & .13 & 0.82 & 0.84 \\
\hline 0.44 & .13 & 0.85 & 0.82 \\
\hline 0.56 & .13 & 1.37 & 1.34 \\
\hline 0.04 & .13 & 0.69 & 0.71 \\
\hline 1.97 & .17 & 1.09 & 1.14 \\
\hline-1.17 & .19 & 1.18 & 2.28 \\
\hline-1.00 & .18 & 1.12 & 1.03 \\
\hline
\end{tabular}

(c) Ediciones Universidad de Salamanca / CC BY-NC-ND

Siglo Cero, vol. 47 (1), n. ${ }^{\circ}$ 257, 2016, enero-marzo, pp. 23-36 
PROPIEDADES PSICOMÉTRICAS DE LA ESCALA GENCAT DE CALIDAD DE VIDA

EN UNA MUESTRA DE PERSONAS SIN HOGAR

EVA MARÍA BENITO HERRÁEZ, CARLES ALSINET MORA Y ARACELI MACIÁ ANTÓN

\begin{tabular}{|c|c|c|c|c|}
\hline 50. Organiza su propia vida. & 0.42 & .13 & 0.79 & 0.74 \\
\hline 51. Elige con quién vivir. & 0.79 & .13 & 0.97 & 0.88 \\
\hline INCLUSIÓN SOCIAL & $\begin{array}{l}\text { Medida } \\
\text { (logits) }\end{array}$ & $\begin{array}{c}\text { Error } \\
\text { estándar }\end{array}$ & Infit & Outfit \\
\hline $\begin{array}{l}\text { 52. Utiliza entornos comunitarios (piscinas públicas, cines, teatros, museos, } \\
\text { bibliotecas...). }\end{array}$ & 1.09 & .22 & 0.58 & 0.63 \\
\hline 53. Su familia le apoya cuando lo necesita. & 1.73 & .26 & 1.07 & 0.77 \\
\hline $\begin{array}{l}\text { 54. Existen barreras físicas, culturales o sociales que dificultan su inclusión } \\
\text { social. }\end{array}$ & 0.17 & .19 & 0.75 & 0.68 \\
\hline $\begin{array}{l}\text { 55. Carece de los apoyos necesarios para participar activamente en la vida } \\
\text { de su comunidad. }\end{array}$ & -0.76 & .17 & 0.97 & 0.91 \\
\hline 56. Sus amigos le apoyan cuando lo necesita. & 0.55 & .20 & 0.87 & 0.98 \\
\hline $\begin{array}{l}\text { 57. El servicio al que acude fomenta su participación en diversas actividades } \\
\text { en la comunidad. }\end{array}$ & -0.43 & .18 & 1.93 & 1.76 \\
\hline 58. Sus amigos se limitan a los que asisten al mismo servicio. & 0.28 & .19 & 0.98 & 1.09 \\
\hline 59. Es rechazado o discriminado por los demás. & 2.62 & .18 & 0.81 & 0.79 \\
\hline DERECHOS & $\begin{array}{l}\text { Medida } \\
\text { (logits) }\end{array}$ & $\begin{array}{l}\text { Error } \\
\text { estándar }\end{array}$ & Infit & Outfit \\
\hline $\begin{array}{l}\text { 60. Su familia vulnera su intimidad (lee su correspondencia, entra sin llamar } \\
\text { a la puerta...). }\end{array}$ & -2.03 & .43 & 3.09 & 2.24 \\
\hline 61. En su entorno es tratado con respeto. & 2.31 & .17 & 0.80 & 0.96 \\
\hline $\begin{array}{l}\text { 62. Dispone de información sobre sus derechos fundamentales como ciu- } \\
\text { dadano. }\end{array}$ & 2.52 & .17 & 0.56 & 0.56 \\
\hline $\begin{array}{l}\text { 63. Muestra dificultades para defender sus derechos cuando éstos son vio- } \\
\text { lados. }\end{array}$ & 1.82 & .17 & 0.87 & 0.82 \\
\hline 64. En el servicio al que acude se respeta su intimidad. & -0.58 & .26 & 1.32 & 0.85 \\
\hline $\begin{array}{l}\text { 65. En el servicio al que acude se respetan sus posesiones y derecho a la } \\
\text { propiedad. }\end{array}$ & -0.45 & .25 & 1.44 & 1.82 \\
\hline $\begin{array}{l}\text { 66. Tiene limitado algún derecho legal (ciudadanía, voto, procesos legales, } \\
\text { respeto a sus creencias, valores, etc.). }\end{array}$ & 0.43 & .20 & 1.41 & 1.07 \\
\hline $\begin{array}{l}\text { 67. En el servicio al que acude se respetan y defienden sus derechos (confi- } \\
\text { dencialidad, información sobre sus derechos como usuario...). }\end{array}$ & -2.73 & .55 & 1.32 & 0.34 \\
\hline 68. El servicio respeta la privacidad de la información. & -2.73 & .55 & 1.32 & 0.34 \\
\hline 69. Sufre situaciones de explotación, violencia o abusos. & 1.43 & .18 & 0.73 & 1.08 \\
\hline
\end{tabular}

El resumen del ajuste global al modelo de las personas y los ítems recogido en la Tabla 1 ofrece media igual a 0 y resultados de infit y outfit cercanos a 1 para los ítems en la mayoría de ellas, destacando Bienestar Emocional e Inclusión Social con un ajuste perfecto. Teniendo en cuenta que los límites de ajuste aceptables propuestos por Linacre (2002) son de 0.5 y 1.5, la dimensión que peor se ajusta al modelo, aunque sin sobrepasar los límites aceptables, es la de los Derechos. En el

(C) Ediciones Universidad de Salamanca / CC BY-NC-ND

Siglo Cero, vol. 47 (1), n. ${ }^{\circ} 257,2016$, enero-marzo, pp. 23-36 
caso de las personas, a pesar que los resultados son menos ajustados que en el caso de los ítems, se consideran aceptables al no superar los límites recomendados pudiendo afirmar que explican los patrones de respuesta de las personas a los ítems de forma adecuada.

Tal y como se recoge en la Tabla 2 sobre los ítems de la escala la mayoría de ellos muestra un ajuste adecuado al modelo. Los que muestran valores superiores a 2.00 se encuentran desajustados y no manifiestan la suficiente sensibilidad para medir a las personas en el continuo, provocando distorsiones importantes en la medición. En la escala sucede en tres de ellos: el 40 (En el servicio al que acude se supervisa la medicación que toma), el 48 (Otras personas deciden cómo gastar su dinero) y el 60 (Su familia vulnera su intimidad [lee su correspondencia, entra sin llamar a la puerta...]). Los errores estándar oscilan entre .13 y .55. La mayoría de los ítems no supera el .20. La dimensión más problemática es la de Derechos, con seis de sus nueve reactivos mostrando valores superiores a .25. Por el contrario, los valores de error más pequeños se encuentran en Autodeterminación: en seis de los nueve ítems el valor es de .13.

La localización de los ítems en el continuo (desde 2.52 a -2.73) permite identificar un amplio rango de resultados en los ámbitos que componen la CV de los participantes y evaluar diferentes niveles de CV para los participantes. La precisión de la medida se ha analizado mediante las funciones de información de los 69 ítems, las ocho dimensiones y la escala total, al determinar la región del continuo donde los ítems son más precisos para cada una de las dimensiones de la escala. Los resultados gráficos obtenidos mediante su análisis manifiestan que la mayor precisión de la medida se obtiene en los niveles más extremos del rasgo evaluado, entre \pm 3 y \pm 4 logits tanto para los ítems de cada dimensión como para la escala.

Una vez comprobado el ajuste de los datos al modelo y la precisión de la medida, es necesario obtener los estadísticos de confiabilidad de los ítems y de las personas según las diferentes dimensiones que componen la escala. En la Tabla 3 se presentan estos resultados.

\begin{tabular}{|c|c|c|c|c|c|c|c|c|c|c|c|c|c|c|c|c|}
\hline \multicolumn{10}{|c|}{ TABLA 3. Estadísticos de fiabilidad y separación de las personas y los ítems } \\
\hline & $\begin{array}{c}\text { Bienestar } \\
\text { Emocional }\end{array}$ & \multicolumn{2}{c|}{$\begin{array}{c}\text { Relaciones } \\
\text { Interpersonales }\end{array}$} & \multicolumn{2}{c|}{$\begin{array}{c}\text { Bienestar } \\
\text { Material }\end{array}$} & \multicolumn{2}{c}{$\begin{array}{c}\text { Desarrollo } \\
\text { Personal }\end{array}$} & \multicolumn{2}{c|}{$\begin{array}{c}\text { Bienestar } \\
\text { Físico }\end{array}$} & \multicolumn{2}{c|}{$\begin{array}{c}\text { Autodetermi- } \\
\text { nación }\end{array}$} & \multicolumn{2}{|c|}{$\begin{array}{c}\text { Inclusión } \\
\text { Social }\end{array}$} & \multicolumn{2}{c|}{ Derechos } \\
\hline & $\mathrm{R}$ & $\mathrm{M}$ & $\mathrm{R}$ & $\mathrm{M}$ & $\mathrm{R}$ & $\mathrm{M}$ & $\mathrm{R}$ & $\mathrm{M}$ & $\mathrm{R}$ & $\mathrm{M}$ & $\mathrm{R}$ & $\mathrm{M}$ & $\mathrm{R}$ & $\mathrm{M}$ & $\mathrm{R}$ & $\mathrm{M}$ \\
\hline $\begin{array}{c}\text { Fiabilidad } \\
\text { (Personas) }\end{array}$ & .83 & .86 & .81 & .83 & .71 & .73 & .79 & .81 & .76 & .79 & .67 & .71 & .71 & .75 & .70 & .75 \\
\hline $\begin{array}{c}\text { Separación } \\
\text { (Personas) }\end{array}$ & 2.22 & 2.46 & 2.06 & 2.24 & 1.57 & 1.65 & 1.92 & 2.08 & 1.76 & 1.94 & 1.43 & 1.58 & 1.57 & 1.75 & 1.54 & 1.73 \\
\hline $\begin{array}{c}\text { Fiabilidad } \\
\text { (Ítems) }\end{array}$ & .97 & .98 & .98 & .98 & .95 & .96 & .95 & .96 & .96 & .97 & .96 & .96 & .97 & .97 & .95 & .97 \\
\hline $\begin{array}{c}\text { Separación } \\
\text { (Ítems) }\end{array}$ & 6.19 & 6.45 & 7.22 & 7.46 & 4.43 & 5.00 & 4.53 & 4.72 & 4.93 & 5.43 & 4.86 & 5.10 & 5.75 & 6.05 & 4.51 & 5.74 \\
\hline
\end{tabular}

Nota: $\mathrm{R}=$ Real; $\mathrm{M}=$ Modelo. 
En todas y cada una de las dimensiones la fiabilidad de los ítems que las componen es alta ( $\geq .95)$, destacando especialmente la de Relaciones Interpersonales con un valor de .98. La fiabilidad de las personas obtiene valores adecuados en casi todas las dimensiones, sobre todo en la de Bienestar Emocional. La que presenta resultados más cuestionables es la de Autodeterminación, que a pesar de todo ofrece una estimación aceptable (.67).

Los valores altos de los índices de separación manifiestan la adecuación de las medidas de las dimensiones de la escala para diferenciar el rasgo latente. Los índices relativos a los ítems para cada una de ellas sugieren que la escala permite discriminar entre diferentes niveles de CV para los sujetos, mientras que los obtenidos para las personas manifiestan que en la muestra la escala discrimina entre los participantes. Todos los resultados tanto de ítems como de personas son superiores a 0.50 , siendo sensiblemente más altos en los ítems y especialmente en las de Relaciones Interpersonales y Bienestar Emocional.

\section{Discusión}

La adecuación del modelo de RSM para analizar las propiedades de una escala medida en intervalos se evidencia en trabajos como el de Arias et al. (2010), quienes analizaron las propiedades psicométricas de la Escala Gencat de Calidad de Vida (Verdugo et al., 2008) para una muestra de personas drogodependientes y el de Gómez, Arias, Verdugo y Navas (2012b) al realizar un profundo estudio sobre las propiedades psicométricas de la Escala INTEGRAL de Calidad de Vida (Verdugo et al., 2009). En ambos casos se analizó previamente la fiabilidad mediante la TCT; sin embargo, el segundo estudio amplió los resultados a la comprobación no sólo de la validez del instrumento, sino también de la posibilidad de analizar el rasgo latente y las categorías de respuesta de la escala entre otros elementos. En el presente estudio los esfuerzos se han concentrado en el análisis del ajuste de los datos al modelo de Rasch y a la exactitud de la medida para los ítems y las personas al pretender ser una primera aproximación al análisis de las propiedades psicométricas de la escala aplicada a una muestra de PSH de la ciudad de Lleida, utilizando para ello el modelo de RSM.

Los resultados obtenidos muestran un buen ajuste al modelo de la mayoría de las dimensiones y de los ítems de cada una de ellas. Es Derechos la que peor comportamiento muestra en relación al ajuste y a los errores de medida ofrecidos. A ello se añade la existencia de un ítem desajustado, por lo que se puede concluir que es la dimensión más problemática de todas.

Tres de los ítems superan los valores permitidos para considerarlos útiles, lo que supone el $4.4 \%$ del total, un porcentaje relativamente bajo. Al comparar los resultados obtenidos con el estudio de Arias et al. (2010) el número de ítems desajustados es sensiblemente menor, siendo siete frente a tres los que superan los límites de 0.5-2.00 basados en Linacre (2002). El más cuestionable es el 60 (Su familia vulnera su intimidad [lee su correspondencia, entra sin llamar a la puerta...]) con 
valores desajustados especialmente en el outfit (3.09), lo que pone de manifiesto que las personas muestran valoraciones extremas en esta situación. El 40 (En el servicio al que acude se supervisa la medicación que toma) también supone la presencia de valores extremos aunque en menor medida que el anterior. No debe extrañar la existencia de estos elementos distorsionadores ya que la situación de exclusión severa que padecen las PSH se manifiesta especialmente en la ruptura grave y traumática de las relaciones familiares, por lo que la presencia de un familiar cercano en las actividades cotidianas carece de todo sentido. Lo mismo sucede en las situaciones de autocuidado: las dificultades que presenta el colectivo para disfrutar de un espacio físico adecuado que permita, por ejemplo, la toma de la medicación diaria imposibilita valorar adecuadamente este aspecto entre otros muchos. Los ítems problemáticos en todo caso deben ser revisados en posteriores análisis y averiguar si su eliminación mejora los resultados o deben ser modificados o transformados para ajustarse a la realidad y la situación de las PSH.

Al analizar la fiabilidad de las dimensiones de la escala, pese a los buenos resultados que ofrecen los estadísticos de la escala y las personas, es preciso puntualizar que es Autodeterminación la que peor comportamiento manifiesta. Al comparar estos resultados con los obtenidos por Arias et al. (2010) la mejora en la fiabilidad de las estimaciones es sensible. La presente investigación manifiesta puntuaciones superiores en todas las dimensiones excepto en Desarrollo Personal, aunque no es una diferencia importante. Cabe destacar el aumento evidente de los resultados en Bienestar Físico y en Autodeterminación respecto a la muestra de personas drogodependientes, especialmente en el primer caso. Si bien es cierto que en lo relativo a la discriminación entre personas el presente estudio mejora los resultados, no supone un hallazgo definitivo ya que sigue habiendo dificultades. Cabría entonces la posibilidad de revisar los ítems con niveles de dificultad más dudosos para mejorar este obstáculo.

Es necesario puntualizar que los análisis realizados son una primera aproximación a su validez y las limitaciones son manifiestas. Una posible explicación de estos resultados puede encontrarse en las propias dificultades del estudio. La más importante de ellas es el tamaño de la muestra: estudios con menos de cien participantes restan confiabilidad y validez a los resultados y, en este caso, los participantes no llegan a esta cifra. Si además se tiene en cuenta la longitud del test, el tamaño muestral supone un hándicap importante. Las posibles soluciones a los obstáculos encontrados en la investigación derivan en ampliar el número de personas de la muestra, replicar la aplicación de la escala a la misma en una o varias ocasiones o incluso en otra muestra de una ciudad con características similares a la de Lleida. Por lo que respecta a la fiabilidad, realizar un análisis más en profundidad sobre los resultados obtenidos puede arrojar más claridad que la obtenida en esta primera aproximación, aunque ampliar el tamaño muestral continuará siendo la mejor opción.

La Escala Gencat de Calidad de Vida ha sido diseñada para evaluar la CV en personas usuarias de servicios sociales en función de la pertenencia a un colectivo concreto, con unas características determinadas y una situación personal, 


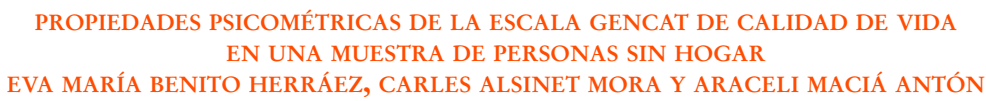

socioeconómica e incluso emocional concreta. Los resultados del presente estudio permiten concluir, pese a las limitaciones observadas, que la escala posee propiedades psicométricas adecuadas para poder evaluar la CV de PSH a pesar de no haber sido diseñada de forma explícita para valorar el constructo en este colectivo concreto, mejorando incluso alguno de los resultados obtenidos para otros que sí pertenecen a los destinatarios de la escala.

\section{Referencias bibliográficas}

Aguirre, B. (2013). El acompañamiento social personalizado como fórmula innovadora de respuesta a personas con trastorno mental y en exclusión social. Zerbitzuan, 54, 127-137.

Arias, B., Gómez, L. E., Verdugo, M. Á. y Navas, P. (2010). Evaluación de la calidad de vida en personas drogodependientes mediante el modelo de Rasch. Revista Española de Drogodependencias, 35 (1), 206-219.

Córdoba-Andrade, L., Gómez-Benito, J. y Verdugo-Alonso, M. Á. (2008). Calidad de vida familiar en personas con discapacidad: un análisis comparativo. Universitas Psychologica, 7 (2), 369-383.

Cummins, R. A. (1997). Assessing the quality of life of adults with profound disabilities. En R. I. Brown. Quality of Life for People with Disabilities. Models, research and practice. United Kingdom: Stanley Thornes.

Cummins, R. A. y Cahill, J. (2000). Avances en la comprensión de la calidad de vida subjetiva. Intervención Psicosocial, 9 (2), 185-198.

Felce, D. y Perry, J. (1995). Quality of life: its definition and measurement. Research in Developmental Disabilities, 16 (1), 51-74.

GómEZ, L. E. (2010). Evaluación de la calidad de vida en servicios sociales: validación y calibración de la Escala GENCAT. (Tesis doctoral sin publicar). INICO, Universidad de Salamanca, Salamanca. Disponible en http://gredos.usal.es/ispui/handle/10366/76489.

Gómez, L. E., Arias, B., Verdugo, M. Á. y Navas, P. (2012a). An outcomes-Based Assessment of Quality of Life in Social Services. Social Indicators Research, 106, 81-93. doi: 10.1007/ s11205-011-9794-9.

Gómez, L. E., Arias, B., Verdugo, M. Á. y Navas, P. (2012b). Application of the Rasch rating scale model to the assessment of quality of life of person with intellectual disability. Journal of Intellectual and Developmental Disability, 37 (29), 114-150. doi: 10.3109/13668250.2012.682647.

Gómez, L. E., Verdugo, M. Á. y Arias, B. (2010). Calidad de vida individual: avances en su conceptualización y retos emergentes en el ámbito de la discapacidad. Behavioral Psychology/Psicología Conductual, 18 (3), 453-472.

Gómez, L. E., Verdugo, M. Á., Arias, B. y Arias, V. (2010). A comparison of alternative models of individual quality of life for social service recipients. Social Indicators Research, 101, 109-126. doi: 10.1007/s11205-010-9639-y.

Linacre, J. M. (2002). What do Infit and Outfit, Mean-square and Standardized mean? Rasch Measurement Transactions, 16 (2), 878.

Linacre, J. M. (2009). Winsteps Rasch Measurement Computer Program. Chicago, EU, www. winsteps.com/index.htm.

Organización Mundial de la Salud (1948). Constitución de la Organización Mundial de la Salud [Documento en línea]. Disponible: http://www.apps.who.int/gb/bd/PDF/bd47/SP/ constitucion-sp.pd?ua=1.

(C) Ediciones Universidad de Salamanca / CC BY-NC-ND

Siglo Cero, vol. 47 (1), n. ${ }^{\circ}$ 257, 2016, enero-marzo, pp. 23-36 
Park, J., Turnbull, A. P. y Turnbull, H. R. (2002). Impacts of Poverty on Quality of Life in Families of Children with Disabilities. Exceptional Children, 68 (2), 151-170.

Petry, K., Maes, B. y Vlaskamp, C. (2005). Domains of Quality of Life of People with Profound Multiple Disabilities: the perspective of Parents and Direct Support Staff. Journal of Applied Research in Intellectual Disabilities, 18 (1), 35-46.

Ruiz, N., Verdugo, M. Á. y Orgaz, M. B. (2011). Análisis de la calidad de vida en trabajadores con discapacidad en situación de exclusión social en empleo ordinario. Documentación Social, 161, 187-201.

Schalock, R., Brown, I., Brown, R., Cummins, R. A., Felce, D., Matikka, L., Keith, K. D. y Parmenter, T. (2002). Conceptualization, Measurement, and Application of Quality of Life for Persons with Intellectual Disabilities: Report of an International Panel of Experts. Mental Retardation, 40 (6), 457-470.

Schalock, R. y Verdugo, M. Á. (2003). Calidad de vida. Manual para profesionales de la educación, salud y servicios sociales. Madrid, España: Alianza Editorial.

Schalock, R. y Verdugo, M. Á. (2007). El concepto de calidad de vida en los servicios y apoyos para personas con discapacidad intelectual. Siglo Cero. Revista Española sobre Discapacidad Intelectual, 38 (4), 21-36.

Schwartzmann, L. (2003). Calidad de vida relacionada con la salud: aspectos conceptuales. Ciencia y Enfermería, IX (2), 9-21.

Shumaker, S. A. y Naughton, M. J. (1995). The international assessment of health-related quality of life: a theroretical perspective. En S. A. SHUMAKER y R. BERZON (Eds.), Quality of life: theory and translation measurement and analysis (pp. 3-18). Oxford, England: Rapid Communication of Oxford.

Verdugo, M. Á., Arias, B., Gómez, L. E. y Schalock, R. L. (2008). Formulari de l'Escala Gencat de Qualitat de vida. Manual d'aplicació de l'Escala Gencat de Qualitat de vida. Departament d'Acció Social i Ciutadania. Generalitat de Catalunya.

Verdugo, M. Á., Arias, B., Gómez, L. E. y Schalock, R. L. (2009). Escala Integral de Calidad de Vida. Madrid: CEPE.

Verdugo, M. Á., Arias, B., Gómez, L. E. y Schalock, R. L. (2010). Development of an objective instrument to assess quality of life in social services: Reliability and validity in Spain. International Journal of Clinical and Health Psychology, 10 (1), 105-123.

Verdugo, M. Á., Borja Jordán de Urríes, F., Orgaz, B., Benito, M. C., Martín-Ingelmo, R., Ruiz, N. y SANTAMARía, M. (2010). Impacto social del programa ECA CAJA MADRID de empleo con apoyo. Salamanca, España: Instituto Universitario de Integración en la Comunidad.

Verdugo, M. Á., Gómez, L. E. y Arias, B. (2009). Evaluación de la calidad de vida en personas mayores. La escala FUMAT. Salamanca, España: INICO.

Verdugo, M. Á., Gómez, L. E., Arias, B., Santamaría, M., Clavero, D. y Tamarit, J. (2013). Escala INICO-FEAPS. Evaluación integral de la calidad de vida de personas con discapacidad intelectual o del desarrollo. Salamanca, España: Universidad de Salamanca, Publicaciones INICO, Colección Herramientas 7/2013.

Verdugo, M. Á., Schalock, K. D., Keith, R. J. y Stancliffe, R. J. (2006). La calidad de vida y su medida: principios y directrices importantes. Siglo Cero. Revista Española sobre Discapacidad Intelectual, 37 (2), 9-25. 


\section{RESEÑAS}

Flórez, J., Garvía, B. y Fernández, R. (2015). Sindrome de Down: neurobiología, neuropsicologia, salud mental. Bases para la intervención en el aprendizaje, la conducta $y$ el bienestar mental. Madrid: CEPE, Fundación Iberoamericana Down21. ISBN 97884-7869-999-5, 527 pp.

Este tratado, elaborado por Jesús Flórez, Beatriz Garvía y Roser Fernández-Oloria y coeditado por la Fundación Iberoamericana Down21 y la editorial CEPE, quiere aportar una visión actualizada de la perspectiva de la neurociencia y la psicología en relación con el síndrome de Down, además de exponer los fundamentos para actuar y las grandes posibilidades de acción para influir sobre la cognición, la conducta y la salud mental.

La trayectoria profesional y las experiencias personales de los autores garantizan esta obra en la que se nos acerca a la realidad del síndrome de Down, con sus situaciones y condiciones positivas y negativas, con la esperanza de derribar los perjuicios sociales que han levantado barreras y creyendo en la capacidad de las personas con síndrome de Down.

A lo largo de 19 capítulos se encuentran investigaciones sobre genética, neurología, psicología, vida adulta, envejecimiento, problemas conductuales, problemas mentales, atención, memoria, lenguaje... que pretenden responder, basándose en el conocimiento de la neurociencia y la psicología, a cuáles son los rasgos comunes de las personas con síndrome de Down siendo conocedores de las diferencias existentes entre los seres humanos. La presencia de un cromosoma 21 extra, con sus más de 500 genes y otro material genético propio de ese cromosoma, introduce una modificación con consecuencias reales sobre la constitución del organismo humano expresada en sus múltiples órganos y sistemas.

Este libro trata de abordar de forma global lo que el cerebro es y significa para poder modificar y realizar una intervención tanto en el aprendizaje como en la conducta y en la salud mental, siendo este triple análisis la novedad de que aporta esta obra.

La primera parte del libro se centra en la realidad del cerebro, su desarrollo y genética, así como la organización de redes neuronales y las consecuencias que produce la trisomía del cromosoma 21, su evolución, cambios o mejora de posibilidades.

En la segunda parte se abordan los temas fundamentales de las funciones cerebrales estudiados por la moderna neuropsicología. En los últimos capítulos se ofrece una visión sobre el bienestar, la construcción de la identidad y la salud mental, deteniéndose en cuáles son los principales trastornos mentales y sus tratamientos psicoterapéuticos y farmacológicos, para apreciar los logros actuales y los futuros retos.

La realidad que se aprecia en las personas con síndrome de Down a lo largo de su vida, la calidad de vida alcanzada en función de sus características personales, de la riqueza de los apoyos recibidos y de los propios esfuerzos supone una apertura de oportunidades para todas las personas. La sociedad no es consciente de la importancia de cambiar actitudes para enriquecer el propio conocimiento, la diferencia es un elemento esencial en los seres humanos por lo que el rechazo que en ocasiones sufren las personas con síndrome de Down es muy probable debido a un desconocimiento del cúmulo de posibilidades de las personas con síndrome de Down. 


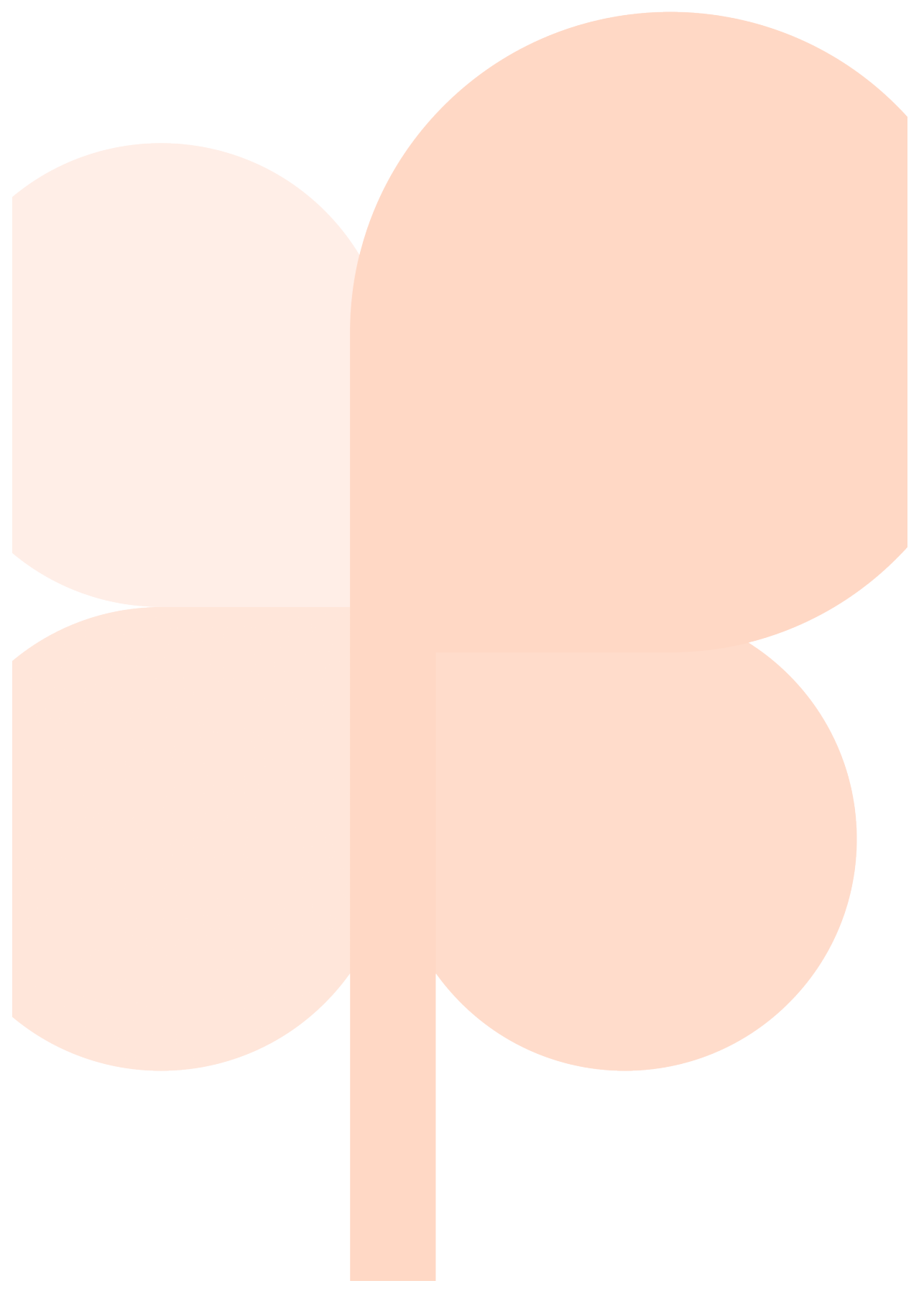

